\title{
An Essay on Synthetic Chemistry of Colloidal Nanocrystals
}

\author{
Xiaogang Peng $(\bowtie)$ \\ Department of Chemistry and Biochemistry, University of Arkansas, Fayetteville, AR 72701, USA \\ Received: 26 March 2009 / Accepted: 6 April 2009 \\ (C)Tsinghua University Press and Springer-Verlag 2009. This article is published with open access at Springerlink.com
}

\begin{abstract}
The central goal of synthetic chemistry of colloidal nanocrystals at present is to discover functional materials. Such functional materials should help mankind to meet the tough challenges brought by the rapid depletion of natural resources and the significant increase of population with higher and higher living standards. With this thought in mind, this essay discusses the basic guidelines for developing this new branch of synthetic chemistry, including rational synthetic strategies, functional performance, and green chemistry principles.
\end{abstract}

\section{KEYWORDS}

Colloidal nancrystal, synthetic chemistry, function materiols, green chemistry, crystallization

\section{Introduction}

The nanotechnology-based industrial revolution, if it is ever realized, will differ from any other industrial revolutions occurring in the last two centuries from a materials viewpoint. From the steam-engine, to electricity, to information technology, every previous industrial revolution was mainly founded on the innovation of physical concepts. However, nanotechnology is so diverse and the materials base, mostly nanomaterials, is so vast and new to mankind. As a result, the main efforts in nanotechnology and nanoscience must be synthesis, manipulation, and processing of nanomaterials, at least in its initial stage.

Nanomaterials refer to numerous types of advanced materials with their physical dimensions in the nanosize regime that often matches the feature sizes associated with the targeted properties. The importance of nanomaterials can also be highlighted by the accelerated consumption of natural resources. Human society in the foreseeable future will always be limited to the earth, a more or less isolated system in the universe except for the seemingly endless photo-radiation from the sun [1]. As the population and living standard increase, we are under increasing pressure to uncover new and innovative means for utilizing all types of raw natural substances as functional materials, preferably with minimum impact to the environment on earth. Although it is too naïve to state that "nano"-materials imply the use of a tiny amount of materials, chemistry developed around synthesis and processing of nanomaterials should certainly offer human society smart pathways to build a much needed harmony with the natural world surrounding us, instead of poisoning our home and that of future generations.

Among all types of nanomaterials, colloidal nanocrystals are probably the largest class at present. Colloidal nanocrystals are nanometer-sized fragments of the corresponding bulk crystals which are typically synthesized and processed as solution species. The properties of colloidal nanocrystals are

Address correspondence to xpeng@uark.edu

\section{Springer}


often found to be size dependent for various reasons. The first well known reason is that their intrinsic physical sizes are comparable to the critical sizes of many important properties of a given class of functional materials [2], such as the wavelength of the electron wavefunction, the diameter of photogenerated excitons, the domain size of magnetic single domains, etc. The second reason is their large surface-to-volume atom ratio, which considerably alters the chemical potential of the structural units in comparison to that for the corresponding bulk crystals [3]. The strongly size-dependent solubility of nanocrystals is a direct result of this property. The third reason is the size dependence of the structure in the nanometer regime, which includes electron band configuration, surface structure and reconstruction, and crystal structure, etc. The unique catalytic properties of gold nanocrystals [4] can be considered as an example of the third type of sizedependent properties. This variety of size-dependent properties coupled with solution-based processability make colloidal nanocrystals a major class of attractive "man-made" materials.

Synthesis of colloidal nanocrystals with rationally controlled size and size distribution is obviously the first step for utilization of their size-dependent properties. It should be pointed out that, although we often talk about size-dependent properties, the reality is that size variations of nanocrystals can be in all three dimensions. Consequently, shape-control of nanocrystals is becoming an important topic in the synthetic chemistry of colloidal nanocrystals.

In addition to rational control over size, shape, size/shape distribution, and other structural aspects of the targeted nanocrystals ("rational" in short), there are two basic rules in judging the significance of a new synthetic development for colloidal nanocrystals. The second rule is the functional performance of the nanocrystals ("functional" in short) because, after all, materials chemists are synthesizing nanomaterials in order to exploit their properties for mankind, instead of merely making an object of beauty. The third rule is green chemistry ("green" in short). Though this last rule has been more or less in everybody's mind, it is still a good idea to spell it out in order to remind scientists in the field constantly. In a certain sense, our generation of scientists has been offered a unique opportunity to reinvent ways for dealing with natural resources, and very likely, only those ones with limited environmental impact will have a future in real life.

Numerous excellent reviews with their focus on synthetic chemistry of colloidal nanocrystals have been published in recent years. Different from these review articles, this essay describes a personal perspective of the field, with its focus on the basic principles for developing the synthetic chemistry of colloidal nanocrystals. For this purpose, I will not limit myself to a given type of colloidal nanocrystals but, as the most promising and most pursued systems, colloidal semiconductor nanocrystals will be used as examples in most cases. Rather than attempting a comprehensive coverage of the relevant literature, a significant portion of the experimental results discussed will be from our own publications.

\section{1. "Rational" synthesis}

Synthesis of colloidal nanocrystals is a combination of solution chemistry and crystal growth. At the moment, it is not clear which one of these two aspects is predominant, and quite possibly, it differs case by case. There is plenty of knowledge about solution chemistry that can foster the development of the synthetic chemistry of colloidal nanocrystals. However, crystallization is not well understood at present. As pointed out repeatedly in the literature, theories on crystallization-nucleation and growth-can both differ by orders of magnitude from the experimental results $[5,6]$. Without a solid foundation in the theory of crystallization, will it be possible for us to develop the necessary rational synthetic chemistry of colloidal nanocrystals? More importantly, such rational syntheses must also follow the other two rules mentioned above, namely "functional" and "green". I will argue that, as the field develops, the answer to this challenging question becomes more and more optimistic. There are several reasons that support this view.

\subsection{The driving force for crystal growth}

The driving force for crystal growth, as pointed out 
by Gibbs, is to minimize the total surface free energy of the system [5]. With the constant specific surface free energy approximation, the total surface free energy of a system is proportional to the total number of surface atoms in the entire system. Ultimately, the Gibbs Law (Eq. (1)) implies that, without kinetic barriers, a crystallization system should result in one single crystal in equilibrium with its saturated solution. In Eq. (1), the sum operation should include every facet area $\left(A_{i}\right)$, with its associated specific surface energy $\sigma_{i}$, on each crystal in the solution.

$\sum G_{\text {surface }}=\sum \sigma_{i} A_{i} \approx \sigma \cdot \sum A_{i}=$ minimum

Equation (1) illustrates that the key thermodynamic parameter controlling a crystallization system is the total surface free energy, which is approximately linearly related to the total surface area. The chemical origin of the surface free energy of a crystal comes from the dangling bonds of the surface atoms. In comparison to the interior atoms, the surface atoms on a crystal are missing at least one nearest neighbor in the lattice. Each of such missing coordination sites on the surface is regarded as a surface dangling bond. Although the surface dangling bonds can be partially compensated by the surface ligands and/or solvent molecules, the free energy difference caused by dangling bonds is quite large in a typical system because the synthesis often requires relatively weak ligands as will be discussed below.

Simple mathematical estimation can show that the surface-to-atom ratio, or the approximate surface free energy contribution to the molar free energy of a crystal, decreases rapidly with the increase in the size of the crystals (Fig. 1) [3]. For a pure substance, the molar free energy is its chemical potential. To further visualize this, let's take $0.2 \mathrm{~nm}$ as the average inter-atom distance in a simple cubic lattice for a crystal with a cubic shape. When the cube edge size is $2 \mathrm{~nm}$, the surface-to-volume atom ratio is about $54.2 \%$, which is very significant. As the cube edge size increases to $20 \mathrm{~nm}$, the surfaceto-volume atom ratio drops sharply to $5.9 \%$. If the edge of the cube further increases to $2 \mu \mathrm{m}$, the surface-to-volume atom ratio will decrease to $0.06 \%$ ! This indicates that, in the typical micron size range where scientists traditionally study crystallization,

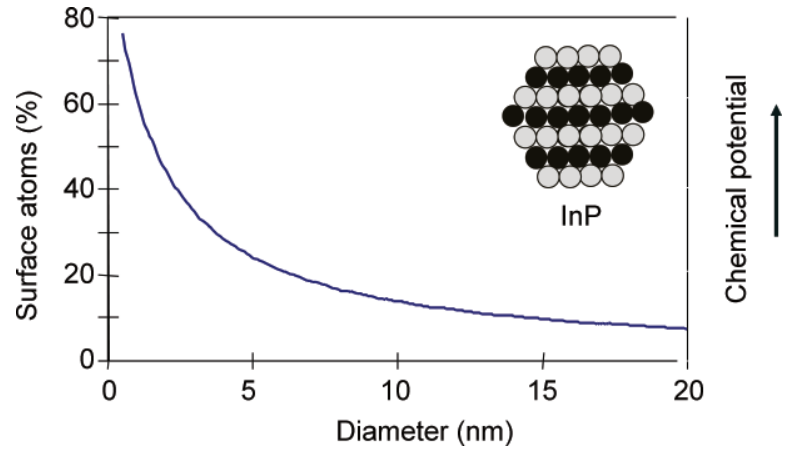

Figure 1 The surface-to-volume atom ratio and chemical potential of nanocrystals calculated using InP lattice parameters

the surface free energy is close to being negligible in a crystal in comparison to the total free energy of the system. Consequently, it becomes very difficult to identify the surface free energy contribution in a crystallization system. This is likely to be one of the main reasons why crystallization has been so difficult to understand in the past.

The above discussions in this sub-section suggest that a fundamental understanding of crystallization may be established by studying crystallization in the nanometer regime. In other words, although we have not reached a satisfactory understanding of crystallization at present, it is very possible that such a success is realistic in the nanometer regime. This will not only provide a necessary foundation for designing rational synthesis for high quality colloidal nanocrystals, but also solve the longstanding challenge to offer a quantitative framework for crystallization in general [7]. Unrelated to the topic but being an interesting perspective, one may argue that understanding crystallization will further impact other fundamental scientific fields, such as phase transitions, biomineralization, surface chemistry in solution, etc.

\subsection{The first set of unique experimental tools for studying formation of colloidal nanocrystals}

The first set of unique experimental tools for studying formation of colloidal nanocrystals originates from the size-dependent properties of colloidal nanocrystals. In fact, the very reason why scientists in the field of crystallization were mostly limited to the micron size range is because of a lack of reliable and convenient tools to probe a crystallization 
system in its initial stage that involves nanometersized clusters/crystals, namely nanoclusters and nanocrystals [5]. Although powerful microscopy techniques with atomic resolution have been continuously developed in the past several decades, such techniques are limited to a small number of crystals in a given set of experiments and often need to be performed under quite restricted conditions. For instance, the formation of "nuclei" (seeds of crystals with sizes of a few nanometers or subnanometer in size) would be very difficult to observe by microscopy studies. In a certain sense, however, one could argue that a crystallization system should be well defined by the initial boundary conditions, which is more or less its nucleation stage.

As mentioned above, there is a broad spectrum of size-dependent properties for colloidal nanocrystals known to the field. Among them, the most convenient ones are the size-dependent optical properties of semiconductor nanocrystals. This is so for several reasons. Firstly, spectroscopic methods became well developed in the last century and widely available in modern laboratories. Secondly, spectroscopic tools are non-invasive in nature and can explore a macroscopic system with ease. Thirdly, the sizedependent optical properties of semiconductor nanocrystals can be readily correlated with the size, shape, and size/shape distribution of a sample with great accuracy [8-10].

The size-dependent optical properties of semiconductors are due to quantum confinement. Detailed discussions of quantum confinement of colloidal semiconductor nanocrystals can be found in a great many publications $[2,11,12]$, and here we will only provide a brief discussion of this phenomenon.

In a piece of a semiconductor, no matter whether it is a bulk crystal or a nanocrystal, the valence electrons are largely delocalized over the entire body, instead of forming localized bonds. This is very much like the large conjugated systems encountered in organic chemistry. When an electron is excited by a photon with the right energy, the electron becomes free to move throughout the entire lattice, except for the fact that the atom losing this electron becomes positively charged (formation of a "hole") and will try to hold the electron through electrostatic interaction. This electrostatic attraction makes the hole follow the electron, which is done by extracting an electron from a neighbor atom in the lattice. Obviously, movement of the hole is more difficult, and thus we say the hole is heavy. Overall, the electron moves rapidly around a slowly moving hole and this photo-generated electron-hole pair is called an exciton. An exciton is similar to a hydrogen atom but the average size of an exciton in semiconductor crystals is much larger than that of a hydrogen atom. This is so because the space between the photo-generated electron and hole is full of other atoms and electrons, instead of being a vacuum as in the case of a hydrogen atom. Typically, the size of an exciton is determined by the dielectric constant of the given semiconductor, the origins of the molecular orbital of the excited state and the ground state, the sizes of the atoms, etc. For example, the Bohr diameter of excitons in a bulk CdSe crystal is approximately $12 \mathrm{~nm}$.

When the physical size of a crystal becomes smaller than the intrinsic size of the corresponding bulk exciton, an exciton is effectively confined inside a box. As a result, just as for a typical particle-in-thebox solution, the energy levels of the exciton (particle) become discrete and the energy separation between the ground state and the first excited state increases markedly as the physical size of the nanocrystal (box) decreases. This is the origin of quantum confinement and quantum size effects in the case of semiconductor nanocrystals. Traditionally, when the size of a semiconductor nanocrystal is within the quantum confinement size regime, we call it a quantum dot.

For typical semiconductors, their bandgaps are in the optically active window. The first absorption peak and photoluminescence (PL) of the excitons lie slightly below the bandgap in a bulk semiconductor due to the contribution of the bonding energy of the excitons (or, the weak electrostatic interaction between the photo-generated electron and hole). Because of quantum confinement, the absorption and PL spectra of quantum dots shift to the blue upon reduction of their sizes. As an example, Fig. 2 (a) shows a series of absorption and PL spectra of nearly monodisperse CdSe nanocrystals in the size range from about $2 \mathrm{~nm}$ to about $10 \mathrm{~nm}$. The lowest excitonic 

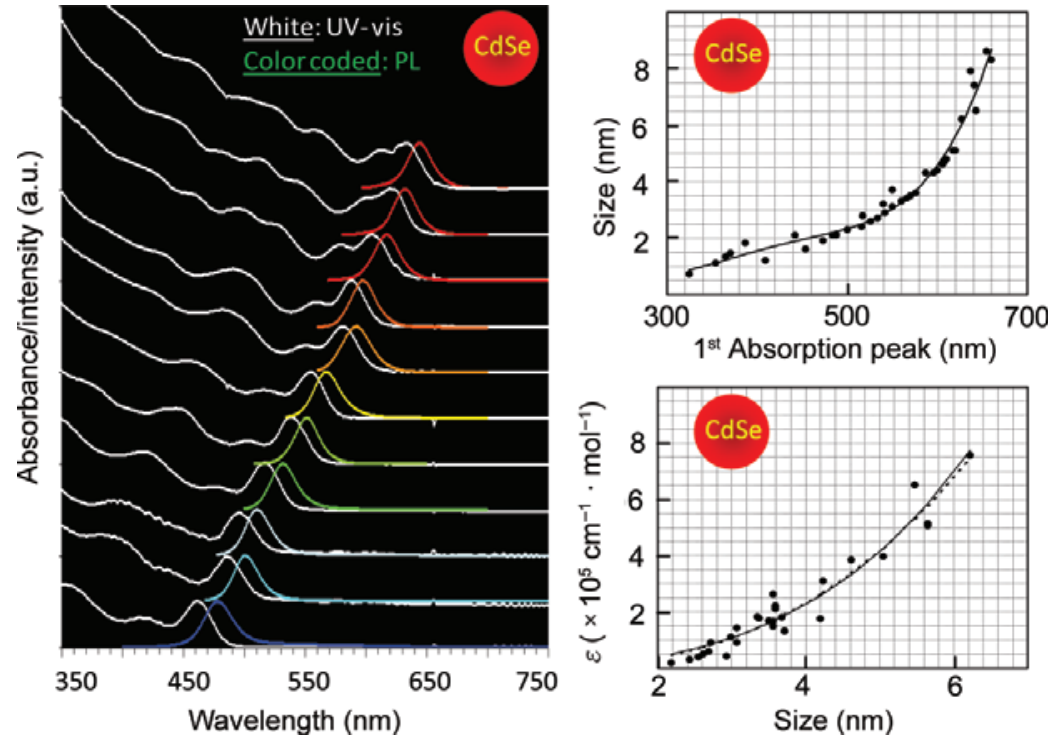

Figure 2 (a) Absorption and photoluminescence (PL) spectra of different sizes of CdSe nanocrystals; (b) plot of size as determined by TEM vs. the first exciton absorption peak; (c) plot of molar extinction coefficient (per mole of particles) vs size of CdSe nanocrystals

absorption peak in each spectrum is determined by the size of the nanocrystals (Fig. 2(b)). The sharp absorption features in each absorption spectrum and narrow peak width of the corresponding PL spectrum confirm that the ensembles of nanocrystals used for recording the spectra were nearly monodisperse. For a nearly monodisperse sample, the particle concentration in the solution can be readily determined by the molar extinction coefficient of the nanocrystals [13] (see Fig. 2 (c) as an example).

For studying crystallization, most nanocrystal samples cannot be truly monodisperse. As a result, it is necessary to extract size distribution information about the nanocrystals from the corresponding optical spectra if one wants to quantitatively define a crystallization system using the spectroscopic method outlined in the above paragraph. If the optical quality of the nanocrystals is decent, the PL of the nanocrystals should only have bandgap emission and it is thus a single peak (Fig. 2(a)). However, because of the uncertainty of the PL quantum yield of the nanocrystals with respect to the different sizes in the ensemble, history of the sample, and environment $[14,15]$, the single-peak feature of the PL spectra can only offer semi-quantitative information about the size distribution profile for a given sample [16].

The absorption spectra of semiconductor nanocrystals, however, always have multiple and overlapping features (Fig. 2(a)). The size distribution information can only be extracted by computer deconvolution of the entire spectrum. However, it is impossible to find one set of standard spectra of truly monodisperse nanocrystals for any type of semiconductor nanocrystals in the literature. The recent deconvolution scheme reported by our group represents a solution to this problem [10]. Instead of using truly monodisperse samples as the reference samples, the UV-vis spectra (see representative ones in Fig. 3(a)) of the best available quality samples of CdS nanocrystals were recorded. Transmission electron microscope (TEM) measurements indicated the particle sizes of these samples had a standard deviation of about $5 \%-7 \%$, which was used to build up a Gaussian distribution for each standard (Fig. 3(b)). By deconvoluting the UV-vis spectra of a sample, a series of contribution factors for the standard spectra were obtained. The corresponding size distribution profile of the test sample could thus be obtained by summing up the product of each contribution factor and the corresponding Gaussian distribution of particle size of the standard sample. As demonstrations, three samples with known size distribution and spectra were examined and comparisons of the expected and simulated results are shown in Figs. 3(c)-(e).

The size, size distribution, and particle concentration of a crystallization system of semiconductor nanocrystals can be determined using the above mathematical scheme coupled with time-resolved spectroscopy techniques. The monomer concentration can be deduced from the difference between the initial absorption value and that in the monomer units in the form of the nanocrystals. Traditionally, size, size distribution, monomer concentration, and particle concentration are considered to be sufficient for defining a crystallization system.

However, as mentioned above, solution chemical processes may be as important as crystallization processes. The evidence in the literature increasingly 

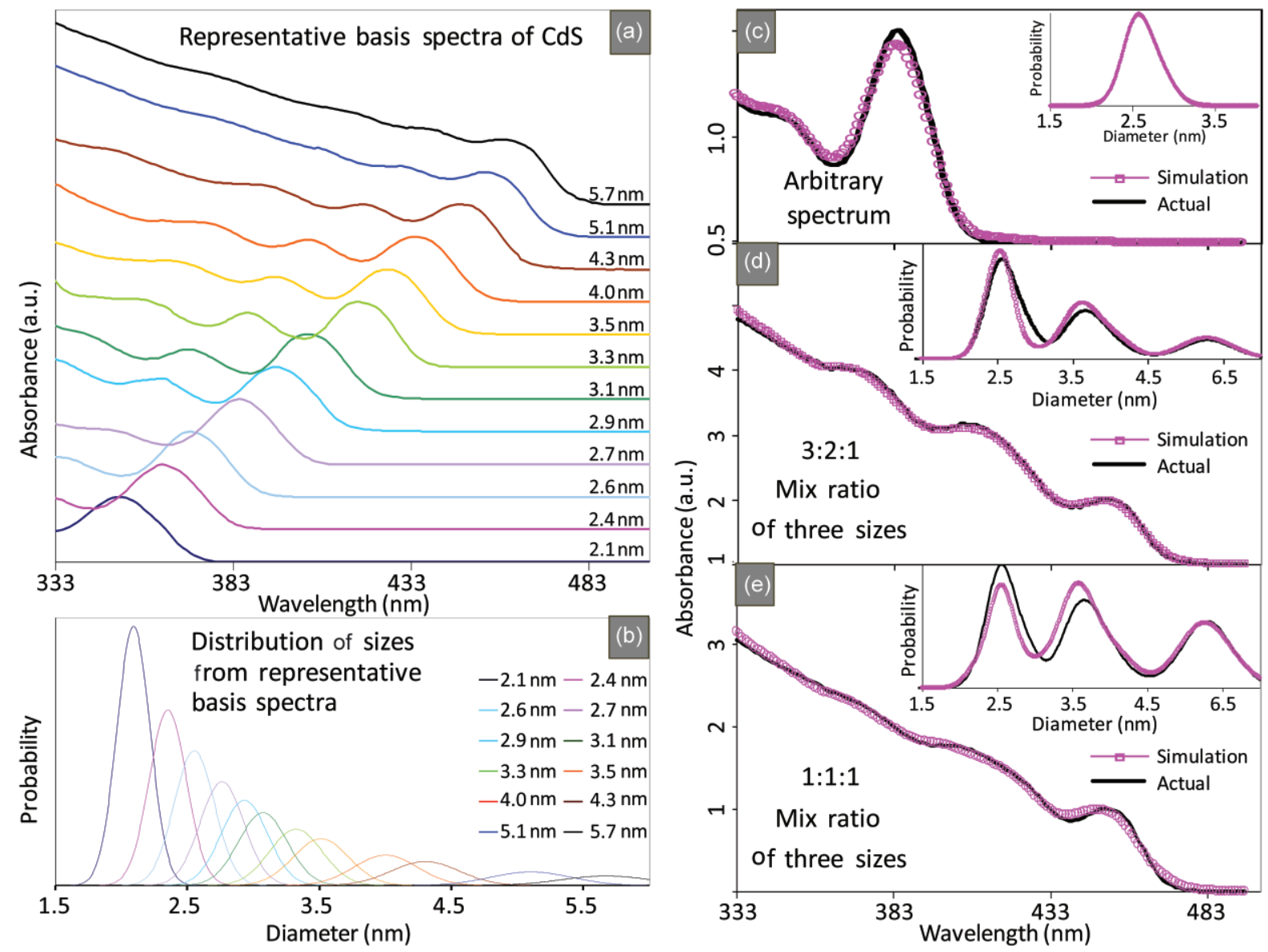

Figure 3 Representative standard spectra (a) and the corresponding size distribution curves (b) of CdS nanocrystals used for spectrum deconvolution. Simulations for three samples with known spectra and size distributions (c), (d), and (e)

indicates that, without a clear molecular reaction mechanism, it is not possible to fully define a crystallization system. For instance, formation of $\mathrm{ZnO}$ nanocrystals at elevated temperatures in nonpolar solvents demonstrated that the nature of the side products actually dictated the size, shape, size/shape distribution, and optical properties of the resulting nanocrystals [17]. Fortunately, such information may be obtained by utilizing another unique feature of colloidal nanocrystals as discussed in the following sub-section.

\subsection{The second set of unique experimental tools for studying the formation of colloidal nanocrystals}

The second set of unique experimental tools for studying the formation of colloidal nanocrystals is based on their solution dispersibility. As a result, many typical solution analytical instruments can often be used to study a crystallization system related to colloidal nanocrystals. This is so because such a crystallization system occurs in solution and often involves a large number of particles and a high concentration of solution species as the starting materials and side products. For instance, the optical spectroscopic method discussed above would be difficult to employ if the crystallization occurred on the surface of a solid substrate, such as the selfassembled quantum dots grown by molecular beam epitaxy, because the number of crystals involved is too small to be detected by conventional absorption spectroscopy.

Common analytical tools used for solution chemistry, especially NMR, UV-vis, and FTIR spectroscopy, can offer information about both nanocrystals themselves and the molecular species in the solution. For example, the unique feature of solution NMR spectroscopy of colloidal nanocrystals is its sensitivity to the difference in chemical 
environment between organic species on the surface and in solution [18]. When an organic species is on the surface of a nanocrystal, the corresponding NMR peaks become considerably broader than those associated with the free species in solution. The broadening due to surface bonding is always strongest for those associated with the atoms next to the bonding sites. For fatty amine and fatty acid ligands, for instance, the NMR peaks associated with the $\alpha-\mathrm{H}$ atoms are the broadest ones in the ${ }^{1} \mathrm{H}$ NMR spectrum (Figs. 4(a) and 4(b)) [19].

The ratio of surface species to solution species is crucial when one studies effects of surface ligands on growth and processing of nanocrystals. As reported recently, dynamic ligand bonding is essential for the growth of an inorganic nanocrystal core surrounded by surface ligands [20]. Therefore, in a growth solution, organic species that can bond to the surface of nanocrystals may very likely exist in two different forms, surface bonded and free in solution. The relative ratio between these two forms reflects the bonding thermodynamics, dynamics, and kinetics. At present, nuclear magnetic resonance NMR is probably the most convenient tool to obtain such information.

It is easy to qualitatively distinguish the surface bonded organic species from the corresponding free
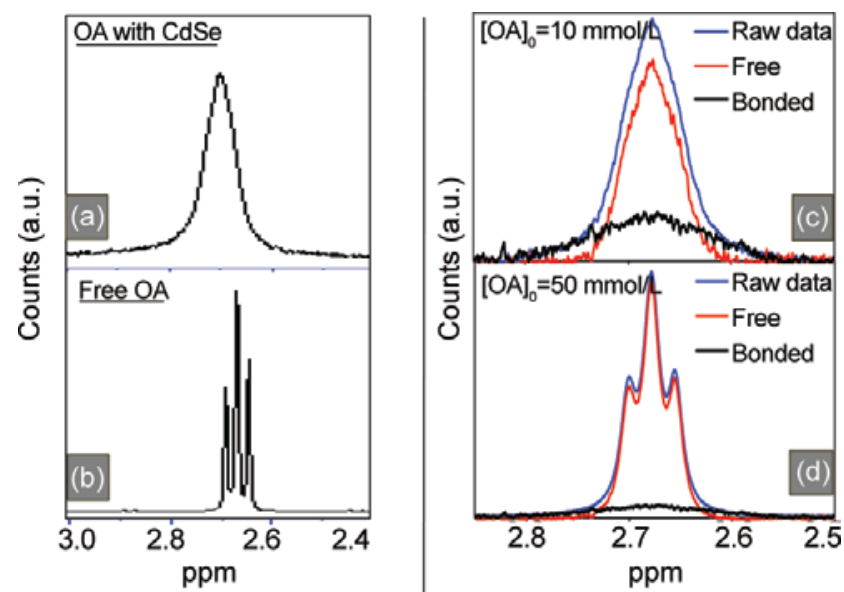

Figure 4 (a),(b) Comparison of the NMR peaks of $\alpha-\mathrm{H}$ atoms in octylamine $(\mathrm{OA})$ in the presence of a high concentration of CdSe nanocrystals (a) and without any CdSe nanocrystals (b); (c),(d) different concentrations ((c) $10 \mathrm{mmol} / \mathrm{L}$ in total, (d) $50 \mathrm{mmol} / \mathrm{L}$ in total) of OA mixed with CdSe nanocrystals. In each case, the raw data (blue) is deconvoluted to a component for free OA (red) and another for surface bonded (black) ones by their substantially different NMR peaks (Figs. $4(\mathrm{a})$ and $4(\mathrm{~b})$ ). When one wants to quantitatively obtain the ratio between the bonded form and free form, it becomes not so straightforward. Experimental results indicate that the peak widths of the bonded and free species may influence each other and vary with their total concentrations. Because ligands can be in fast dynamic exchange between the two forms, the "free" ligands in the solution may become not so free, and a significant portion may experience an environment somewhat associated with the surface. As a result, the NMR signals of formally not-bonded ligands can be significantly broadened [19] (Figs. 4(c) and $4(\mathrm{~d}))$.

A method was introduced to deconvolute the NMR spectra of ligands based on the above hypothesis. The NMR spectrum of a solution with a very low concentration of ligands, just enough for dispersion of the nanocrystals, was recorded and used as the standard spectrum of the bonded ligands. This standard spectrum was found to be extremely broad (the black lines in Figs. 4(c) and 4(d)) and its contribution to the raw data (the blue lines in Figs. 4(c) and 4(d)) for any given sample could be readily extracted. The residual spectrum should thus be the contribution from the not-bonded ligands (the red lines in Figs. 4(c) and 4(d)). The results obtained with this method were found to be quantitatively consistent with the results obtained using solution photoluminescence measurements [19].

Synthesis of high quality colloidal nanocrystals has been mostly carried out in organic solvents. The "greenest" metal precursors are likely to be metal fatty acid salts in most cases (see more discussion later). As a result, FTIR spectroscopy becomes a very powerful tool to monitor the chemical processes accompanying the formation of the nanocrystals. During the formation of the nanocrystals, the metal carboxylate anions are consumed and converted to fatty acids, esters, ketones, amides, etc. In all of these compounds, the carbonyl vibration bands, in the range between 1500 and $1800 \mathrm{~cm}^{-1}$, are very strong and also sensitive to their local structure [17]. In addition, this IR window does not overlap with other strong IR bands. These features make FTIR a not only convenient but also quantitative way to study 
chemical processes involved in the formation of high quality nanocrystals [21].

UV-vis absorption spectroscopy has also been used to study solution chemical processes associated with the formation of high quality nanocrystals. However, unlike FTIR and NMR, strong UV-vis absorption is not very common for solution molecular species. In addition, nanocrystals often possess strong absorption in the common absorption window (see Fig. 2 as an example). As a result, it can be only used in special cases where metal ions form complexes in solution with substantial molar extinction coefficients, such as in the case of gold complexes involved in the formation of gold nanocrystals using the citrate reduction approach [22].

\subsection{The current status of rational synthesis of colloidal nanocrystals}

The current status of rational synthesis of colloidal nanocrystals is substantially advanced in comparison to that in the early stages of development of the field. They may divide its history into two main stages. The first stage represents the time period when scientists working in the field mostly felt that formation of monodisperse nanocrystals was a "black art" which would be very difficult to understand, and thus rational synthesis of colloidal nanocrystals with tightly controlled size/shape distribution, single crystallinity, and desired functions would be effectively prohibited. This early stage ended approximately in the middle 1990s, and the field moved into the current stage, in which scientists feel that rational synthesis can be done at least in a qualitative manner and in some cases quantitative understanding of a synthetic system is actually becoming possible.

The division between the two stages of course is not absolute. Let's have a close look at the most pursued type of colloidal nanocrystals, semiconductor ones. In the early stage, scientists synthesized nearly all types of colloidal semiconductor nanocrystals and started to study many of their interesting properties. However, until the introduction of the famous "organometallic synthesis in hot coordinating solvents" for CdSe nanocrystals by the Brus group [23] and Bawendi's group [24], it was not possible to synthesize any type of semiconductor nanocrystals with a quality as high as those presented in Fig. 2. Although the success was largely limited to CdSe, it must be regarded as a milestone step in the development of the synthetic chemistry of high quality colloidal nanocrystals. This system offered the basis for formulating the concept "focusing of size distribution" in 1998 by the Alivistos group [16], which may be considered as the starting point of rational synthesis of colloidal nanocrystals, and explains why nearly monodisperse nanocrystals can be directly formed in synthesis. This concept is based on the strong size-dependent solubility of nanocrystals in solution. As stated by the Gibbs law (Eq. (1)) and in Fig. 1, the chemical potential of nanocrystals increases dramatically as their size decreases because their surface-to-volume atom ratio increases substantially. Consequently, the solubility of the nanocrystals increases exponentially as the size of the nanocrystals decreases, which can be illustrated by the GibbsThompson equation (Eq. (2)).

$$
S_{d}=S_{\infty} \exp \left(4 \sigma V_{\mathrm{m}} / d R T\right)
$$

Where, $\sigma$ and $V_{\mathrm{m}}$ are the specific surface free energy and molar volume of a crystal, respectively, $S_{\infty}$ is the solubility of the bulk crystal and $S_{d}$ is the solubility for a crystal with a diameter $d, R$ is the gas constant and $T$ is the absolute temperature.

In an ensemble of nanocrystals in solution, if the monomer concentration in the solution is higher than the solubility of all nanocrystals, all nanocrystals will grow. If nucleation is stopped at this moment by varying the reaction conditions, such as reducing the monomer concentration and/or temperature, the size distribution of the ensemble will be reduced rapidly [25]. This is so because the rate of size growth for a nanocrystal decreases proportionally to the square of its size, provided the growth process is diffusioncontrolled. Even if the growth is surface reactioncontrolled, the size growth rate should still increase linearly as the size of the nanocrystals decreases. Conversely, if the monomer concentration in the solution depletes to a level lower than the solubility of the relatively small nanocrystals in the ensemble, they start to shrink in size but the larger ones in the ensemble will continue to grow. Thus, this 
broadens the size distribution of the nanocrystals, namely "defocusing of size distribution". This pair of concepts, "focusing of size distribution" and "defocusing of size distribution", has offered a general guideline for designing rational synthetic schemes for nearly monodisperse nanocrystals over the past ten years.

One may rightfully argue that "focusing of size distribution" does not provide any new insight into controlling the size distribution of a colloidal particle system, since its roots are well discussed in the classical colloid literature [25]. However, the recent discovery of "self-focusing" [10, 21] seems to be a new concept; the term was coined after the observation of direct monomer diffusion to one particle from the surrounding particles when their diffusion spheres are overlapping with each other [10]. In terms of driving force, "self-focusing" is the solubility gradient determined by the solubility difference between the adjacent nanocrystals (see Eq. (2)). Figure 5 schematically illustrates both "focusing of size distribution" and "self-focusing". It should be pointed out that, although "self-focusing" was only discovered very recently, it has already been observed in several different colloidal nanocrystal systems [10, 21, 26]. Furthermore, some of the mysterious results in the existing literature [27-29] can also be well explained by this new concept.

One interesting implication of "self-focusing" is that the definition of the end point of the nucleation process in the classical literature may need to be revisited. The results clearly indicate that "selffocusing" can reduce the particle concentration

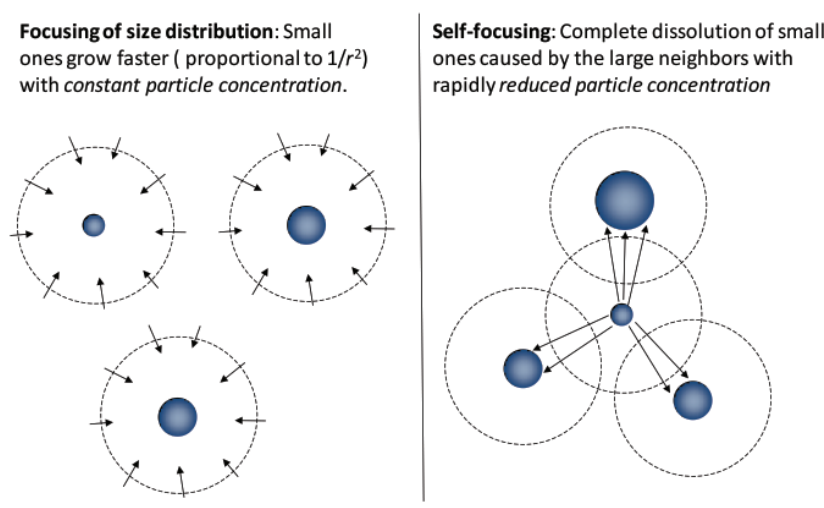

Figure 5 Schematic illustration of "focusing of size distribution" (only for the diffusion-controlled case) and "self-focusing" drastically, with as much as $90 \%$ reduction within roughly two minutes for one specific case [26]. This greatly blurs the division between nucleation and growth-without-nucleation stages discussed in classical crystallization and colloidal sciences, such as the famous Lamar diagram [30]. Furthermore, under "self-focusing" conditions with a high particle concentration, the thermodynamic stability of a cluster/nanocrystal is no longer solely determined by its size and the monomer concentration in the solution. Instead, it is actually more closely related to the particle concentration and the size distribution of the particles in the solution. Consequently, the definition of the critical nucleus size [5] loses its thermodynamic basis. Both implications discussed in this paragraph require us to reconsider the basic picture of nucleation, the very key step defining a crystallization system.

There have been some other conceptual developments in the field of synthetic chemistry of colloidal nanocrystals, which have paved the way for the establishment of today's status of the field. In a single essay, it is impossible to discuss all of them exhaustively. A few of them are briefly described here as examples. Oriented attachment [31-34] has been proposed for the synthesis of non-equilibrium shaped nanocrystals. The noncoordinating solvent approach [35] was developed for tuning the activity of monomers to maintain the necessary balance between nucleation and growth for the formation of monodisperse nanocrystals. Finally, intra-particle ripening [36] was suggested as a way of understanding the shape evolution of elongated nanocrystals into dot-shaped ones. These and other new concepts have not only laid down a decent foundation for the rational synthesis of high quality colloidal nanocrystals but also contributed significantly to our understanding of crystallization in general.

Studies of mechanisms of formation of high quality colloidal nanocrystals have been moving rapidly from qualitative/semi-quantitative to quantitative efforts. Another change is that the chemical reactions involved in the formation of nanocrystals have been attracting more and more attention, instead of the previous emphasis

\section{国 恝 Springer}


on crystallization kinetics. However, there is no single system for the formation of high quality nanocrystals that has been completely and quantitatively defined, at least not at a level generally accepted by the field.

\section{2. "Functional" Materials}

The ultimate goal of synthetic chemistry of colloidal nanocrystals is to develop novel materials which can satisfy the needs of humans currently living on the earth and many generations to come. Obviously, our generation is already far away from the "old" lifestyle, relying mostly on natural materials. As natural resources become rapidly exhausted and the population and overall living standards on the earth increase steadily, it is not difficult to imagine that future generations will rely more and more on "manmade" materials. The key selection rule for new materials is that their function must satisfy the needs of mankind. Such impact should preferably be in the near future because the current situation on the earth is already very tough.

\subsection{The combination of solid-state properties and solution dispersibility}

The combination of solid-state properties and solution dispersibility is a very interesting feature of colloidal nanocrystals in terms of their function (Fig. 6). Solutions and solvents are the natural gifts of the earth's environment to human beings. At very least one can state that, without solution, chemistry would become much less versatile and life on earth would be difficult to imagine. On the other hand, crystals often possess some special properties which may not be available to regular solution species. Colloidal nanocrystals serve as a unique vehicle to bring the functions of crystals into solution. From a viewpoint of chemistry, biology, and medical science, one may immediately realize that colloidal nanocrystals offer the field a huge class of new "substances" with otherwise-not-available functions. From a pure engineering materials viewpoint, colloidal nanocrystals have a general function that is not available to their corresponding bulk crystals i.e., solution processibility and solution compatibility.

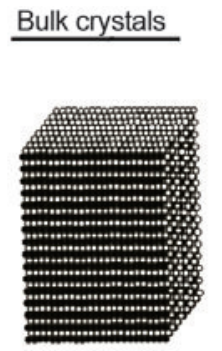
but not in solution
Solid-state functions

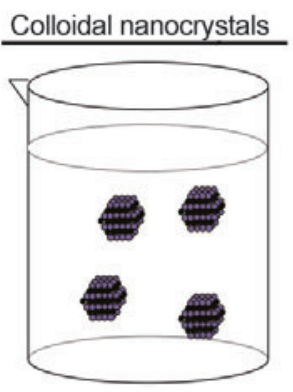

Solution species plus solid-state function

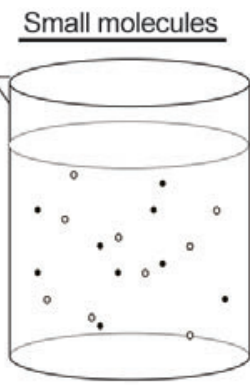

Solution species but no solid-state function
Figure 6 Comparison of bulk crystals, colloidal nanocrystals, and small molecules in terms of solid-state functions and solution compatibility/processability

Although colloidal nanocrystals are expected to have such a magic combination of solutionsolid hybridized functions, it is not a trivial task to realize this unique feature. Because of their substantial surface free energy (Fig. 1) and extremely strong inter-particle interactions due to their large particle mass, colloidal nanocrystals are all metastable species in solution in the absence of some special stabilization mechanisms, such as surface coordination with ligands, surface adsorption of surfactants, or adsorption of charged species. Among all of these means of stabilization, surface coordination of organic ligands is the most robust one and has thus been broadly adopted in the field. At present, the key challenge for realization of the solution-solid hybridized functions is lack of knowledge about the ligand chemistry of colloidal nanocrystals.

It is interesting to notice that solution dispersibility is sometimes a necessary prerequisite to realize the solid-state properties of colloidal nanocrystals. As discussed above, solution processability relies on the surface coordination of organic ligands. Solidstate properties, however, may also depend on the surface bonding of the organic ligands. For example, our recent results quantitatively indicate that the photoluminescence of CdSe semiconductor nanocrystals - a typical solid-state functionincreases linearly with the surface coverage of amine ligands [19] (Fig. 7). Furthermore, even if only some slight aggregation of CdSe nanocrystals-poor solution compatibility-occurred in the solution, both peak position and emission intensity of the 
photoluminescence of the nanocrystals varied significantly.

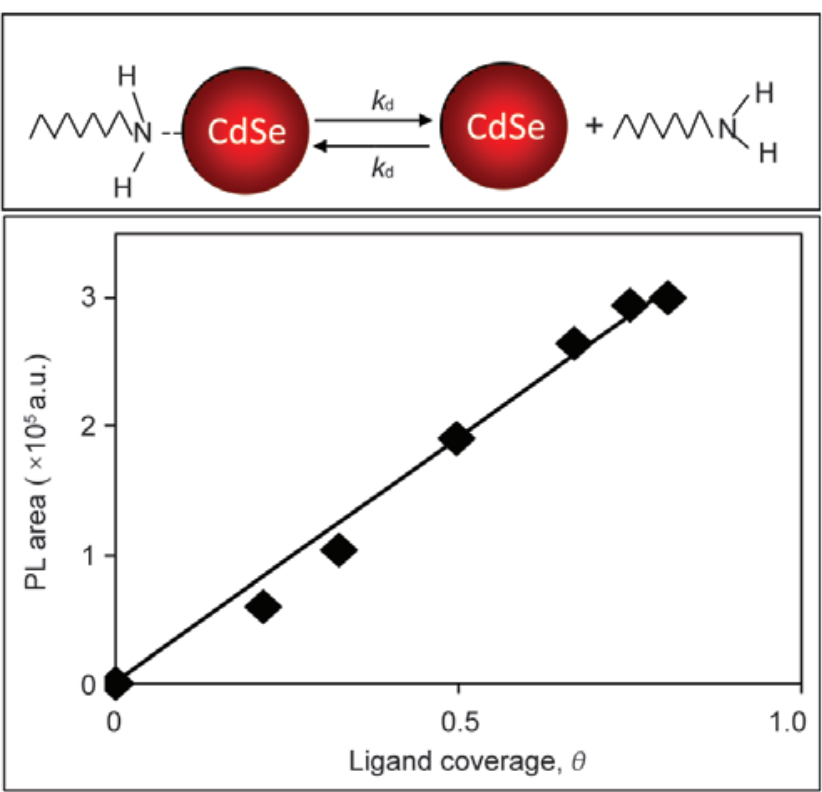

Figure 7 (a)Schematic illustration of surface ligand dynamics; (b) plot of photoluminescence (PL) intensity vs the amine surface ligand coverage of CdSe nanocrystals

The coupling of solid-state function and solution compatibility is often an issue to be considered if the targeted applications are solution based. For example, biomedical labeling $[37,38]$ using colloidal semiconductor nanocrystals based on their strong and size-dependent photoluminescence (Fig. 2) has been extensively explored in the past ten years. Although some success has been demonstrated, there is still huge room for improvement. The main challenges have been noted as the biocompatibility and physiological stability of the nanocrystals. Without proper ligand chemistry, nanocrystals may aggregate, lose their photoluminescence properties, not possess the required circulation lifetime in the body, be trapped through non-specific bonding, and be toxic to tissues, etc.

For applications in the solid state, there are also plenty of results to illustrate the importance of solution compatibility and processability for realization of targeted solid-state functions. For examples, when semiconductor nanocrystals are applied in the fabrication of semiconductor nanocrystal-semiconducting polymer solar cells, the solution properties of the nanocrystals must be carefully controlled [39]. If the nanocrystals cannot be dispersed well in the solution of the semiconducting polymer, it will not be possible to fabricate the devices using solution based approaches. However, the nanocrystals must be incorporated as "naked" particles in the devices after fabrication. This is because any organic ligands on the surface of the nanocrystals would greatly hinder the charge transport in the final devices. The current solution to this delicate balance is to stabilize the nanocrystals with very weak and volatile organic ligands, such as pyridine for $I I-I V$ and $I I I-V$ semiconductor nanocrystals. These weak ligands readily evaporate from the solid film in an inert atmosphere environment, or more efficiently, under vacuum [39].

Directly related to this essay, the solution properties of colloidal nanocrystals are actually extremely important in designing their synthetic chemistry. At one extreme, if the ligands can form extremely strong bonds with the surface atoms on nanocrystals, the nanocrystals will tend to dissolve giving molecular species. Conversely, if the ligandnanocrystal interaction is not sufficiently strong, aggregation will occur, which in most cases defeats rational synthesis. The ideal case is that the ligands bond to the surface atoms in an intermittent fashion, meaning they are dynamically "on and off" during the growth of the nanocrystals. This hypothesis, dynamic bonding of the ligands on the surface of colloidal nanocrystals, has already been demonstrated for some simple systems [19, 20] (see Fig. 7 (b) as an example). For an appreciable growth rate, this dynamic exchange should be reasonably fast thus allowing monomers to reach the surface of the nanocrystals and growth to occur. However, such fast dynamics may greatly damage the function of the nanocrystals, including their solution properties. An interesting way to meet this challenge is to perform the synthesis at elevated temperatures, a temperature sufficiently higher than the temperature for applications, which leads to different ligand dynamics under synthetic conditions and application conditions.

It is clear that ligand chemistry is the key to fully capture the unique solution-solid hybridized 
properties of colloidal nanocrystals. It is not difficult to imagine that such surface coordination is substantially different from the corresponding free ion-ligand coordination in solution. Limited data for bonding kinetics and thermodynamics of surfaces have been reported so far [19, 40,41]. Very little is known at a molecular level, such as bond lengths and bonding configurations. The challenge in extracting such molecular information is that we actually don't have much knowledge of the surface structure of the nanocrystals. It is generally agreed, however, that the surface molecular structure of colloidal nanocrystals may differ from one particle to another in an ensemble. Worse than this, unless all particles in the sample have identical surface structure, there is no reliable technique that can experimentally determine the molecular structure of nanocrystals coated with organic ligands.

\subsection{The extremely large surface-to-volume atom ratio, or specific surface area}

The extremely large surface-to-volume atom ratio, or specific surface area is another general unique property of colloidal nanocrystals. As shown in Fig. 1 , in the nanometer size regime, a crystal can easily possess $10 \%-50 \%$ of surface atoms. As a result, those functions of bulk crystals that depend on the surface should be considerably enhanced by using colloidal nanocrystals. Surface catalysis and surface sensing are two such examples.
The reality, however, is not so rosy. The main problem is that so-called "high quality" colloidal nanocrystals available today are actually "very low quality" in terms of realizing most surfacerelated functions. Taking surface catalysis with noble metal nanocrystals as an example, one can readily illustrate this phenomenon. Many noble metal and transition metal surfaces are well known and widely used surface catalysts in research laboratories and industry. Experimental evidence clearly reveals that, even for the same type of metal, different surfaces may have different catalytic activities. Furthermore, if a chemical species is adsorbed on the surface, especially if chemisorbed, the catalytic functions of the metal will change drastically. Two effects are reponsible for this. The first effect is the alteration of the electronic properties of the surface of the metal. The second effect is the blocking of the surface catalytic sites. In these two aspects, the structure of "high quality" colloidal nanocrystals reported in the literature is generally poor as far as surface catalytic applications are concerned.

Figure 8 (a) illustrates a common type of "high quality" colloidal nanocrystal with organic ligands coordinated to its surface during the synthesis. According to the two structural criteria required for surface catalysis described in the above paragraph, such nanocrystals are far from ideal. Firstly, the steric blocking of the surface monolayer is an obvious issue. There are some interesting developments

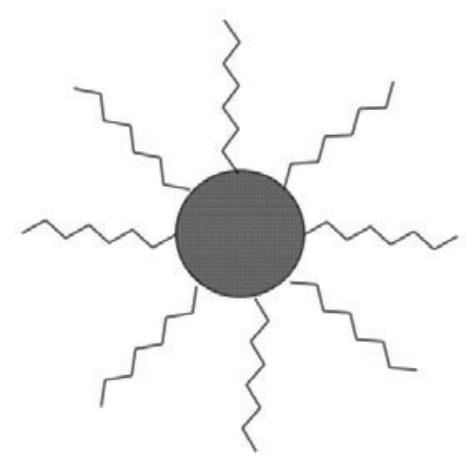

Common colloidal nanocrystals coordinated with traditional ligands

(a)

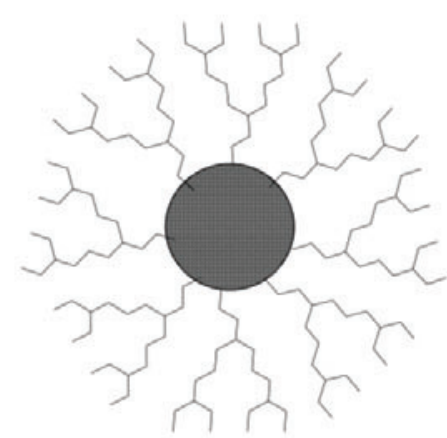

One type of "advanced" colloidal nanocrystals (dendron-nanocrystals)

(b)

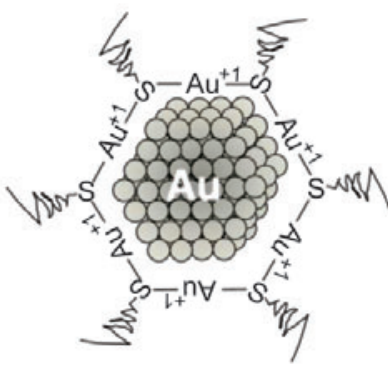

Au nanocrystal with possible surface atom-ligand shell

(c)

Figure 8 Colloidal nanocrystals with their ligands schematically shown (a) with conventional ligands; (b) coated with one type of novel ligand). The cartoon (c) shows a gold nanocrystal with its possible surface atom-ligand shell with organic thiols as its ligands. Such colloidal nanocrystals show very poor accessibility to the surface sites and non-zero valence of the surface sites which is undesirable for catalysis 
in the field involving the use of new ligands for stabilizing colloidal nanocrystals, often through ligand replacement after synthesis. However, nearly all of these efforts yield nanocrystals with a "better" protective coating, with tighter surface bonding and/ or denser surface packing in the ligand monolayer in comparison with traditional ligands (one example [42] is shown in Fig. 8 (b)). Thus, all of these efforts to modify ligand chemistry are working against any applications using the surface of the colloidal nanocrystals as functional sites, such as for surface catalysis or sensing.

Secondly, there is some clear evidence that indicates that the surface sites of colloidal nanocrystals may differ substantially from those in bulk crystals. Thus, even without considering ligand blocking, colloidal nanocrystals are not suited for surface catalysis, at least not by the same catalytic mechanism as their corresponding bulk counterparts. For example, experimental results and theoretical simulations all indicate that the exposed surface gold sites in gold nanoclusters coated with thiol ligands are not neutral gold atoms $\left(\mathrm{Au}^{0}\right)$ as they are for the bulk crystals. Instead, these surface gold sites are gold ions $\left(\mathrm{Au}^{+}\right)$[43]. Although these results were only obtained for nanoclusters which are at the small size end for colloidal gold nanocrystals, the evidence suggests that such surface structure features (schematically shown in Fig. 8(c)) might also apply to larger sizes of gold nanocrystals coated with thiol ligands. When placing thiol ligands onto the surface of gold nanocrystals, it was found that the solution needs to be basic in order to achieve a reasonable surface ligand coverage [44]; the basic conditions are needed to deprotonate the thiols, giving negatively charged thiolates. Usually, each nanocrystal has several tens to hundreds of thiolate ligands depending on its size. If the surface gold atoms were not positively charged, each thiolatecoated gold nanocrystal would become an extremely negatively charged species, with several tens to several hundreds of elemental charges. This is clearly unreasonable in a solution environment.

Overall, a substantial surface reconstruction (Fig. 8(c)) and surface steric effects (Fig. 8) are both present in colloidal nanocrystals dissolved in solution.
There are currently barely any efforts to solve these problems, which makes it very difficult to fully exploit the surface functions of colloidal nanocrystals in solution. One may speculate that such a challenge will require some clever design of a monolayer of surface ligands on a nanocrystal, which will require substantial knowledge of the surface structure of the nanocrystals as well as the configuration and dynamics of the ligand monolayer.

\subsection{Size-dependent properties of colloidal nanocrystals}

Size-dependent properties of colloidal nanocrystals are their most interesting properties, although the solution features and high specific surface area discussed above are important as well. Colloidal nanocrystals can have a very diverse spectrum of composition. In principle, one can make any type of crystal into colloidal nanocrystals with appropriate choice of solvent. This, on the one hand, implies a great opportunity to obtain unique functional materials, which can be altered and controlled by simply varying the size of the corresponding colloidal nanocrystals. On the other hand, it offers synthetic chemists working in the field of colloidal nanocrystals a more or less endless list of synthetic targets. Indeed, there is some momentum in the field that aims towards making colloidal nanocrystals with all possible compositions. The main efforts in the field, however, are concentrated in a few classes of colloidal nanocrystals which have the most promise for impact on human life in the foreseeable future. While being not completely against surveying all possible compositions of colloidal nanocrystals allowed by nature, I would strongly support the idea that synthesis of colloidal nanocrystals should either target some well defined functions or yield fundamental knowledge for science. The research space is simply too large and the time is too tight to allow us to spread our efforts randomly.

The most explored colloidal nanocrystals at present are inorganic semiconductor ones, or quantum dots. The most targeted function of quantum dots is their-size dependent emissive properties (Fig. 2). This is not very surprising. For the earth, solar radiation is the only external input and 
having evolved in such an environment, mankind is dependent on light. As a result, light emitting and emissive materials are crucial for life. Although people have been lighting up the earth for thousands of years, the commonly seen lighting devices in our modern home - incandescent light bulbs - have a power efficiency of only $\sim 2 \%$ on average (conversion efficiency from electric power to light power). Human beings use more than $20 \%$ of all energy consumption in lighting. Thus, if one can improve the power efficiency of our light bulbs to $50 \%$ (United States DoE target for year 2015), we would automatically reduce our total power consumption by about $20 \%$. The most promising technology at present is the solid-state lighting (SSL) technology based on light-emitting diodes (LEDs) and downconversion emissive materials, such as inorganic phosphors, semiconductor nanocrystals, etc.

In addition to the lighting industry, there are so many other urgent needs for high performance emissive materials. Examples include lasers, biomedical labeling reagents, displays, high efficiency and low cost LEDs, and sensing. It should be pointed out that there are some competitive emissive materials available, such as inorganic phosphors and organic dyes, and semiconductor nanocrystals are unlikely to replace all of these. In the foreseeable future, the applications of semiconductor nanocrystals should be those that are difficult to realize with other competitive materials.

Let's use the development of ideal emissive semiconductor nanocrystals as the example to illustrate the importance of considering "function" as one of three guideline rules in the synthetic chemistry of colloidal nanocrystals. If one takes the main targeted function of semiconductor nanocrystals as high performance emissive materials, the first concern of the synthetic chemistry is the function of the dots measured against the requirements / expectation as the emitters. Control over the size and size distribution is important in most cases because it dictates the emission color and color purity. As shown in Fig. 2, when the size of semiconductor nanocrystals varies, the colors of their intrinsic bandgap photoluminescence change drastically. This offers a good reason for scientists to pay close attention to controlling both the size and size distribution of semiconductor nanocrystals.

The most critical parameter as emitters, however, is their emission efficiency. This means that, judged by their function, a "good looking" sample under TEM may not be a high quality emitter at all. Even in the early stages of the synthetic chemistry of semiconductor nanocrystals, this functional criterion was actually employed in helping to choose synthetic routes.

In the 1980s, there were several different more or less equally explored synthetic methods for semiconductor nanocrystals, namely synthesis in aqueous solution, synthesis in organic media, and synthesis using mixed aqueous/nonpolar solvents with soft templates (such as reverse micelles, microemulsions, etc.). Among these approaches, the last one probably yielded semiconductor nanocrystals with the best control over size and size distribution. However, the field eventually moved into synthesis in organic solvents with high boiling points, which was mostly promoted by Louis Brus and his collaborators/students [45]. At that time, although none of these methods yielded nanocrystals with good emissive properties, such a choice was justified by the fundamental hypothesis that highly emissive nanocrystals must be highly crystalline. Consequently, a high temperature synthesis should be ideal for synthesizing such nanocrystals because general wisdom teaches us that high quality single crystals are easier to obtain at high temperatures. With such a hypothesis, one was not surprised to see the movement towards solvents with higher and higher boiling points pioneered by the Brus group [23, $24,46]$.

The synthesis of highly emissive semiconductor nanocrystals (not including core/shell ones) reached its high mark in 2002 [14] (Fig. 9), and was indeed realized in an organic solvent at a quite high temperature, about $300{ }^{\circ} \mathrm{C}$. This took more than ten years after the high temperature approach was coined by the Brus group [23]. After various modifications of this high temperature approach, it was gradually recognized that it is necessary to have a desired surface structure (reconstruction) besides a single crystalline core in order to reach high 

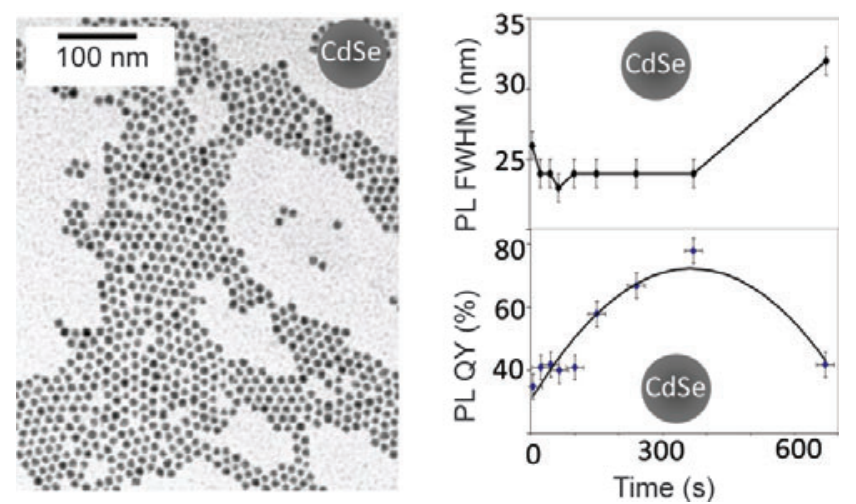

Figure 9 Photoluminescence peak width (top right) and quantum yield (bottom right) of CdSe nanocrystals at different growth times. The size distribution of all these samples was quite close to each other (see a representative TEM image at left) although their PL quantum yields (QY) showed substantial differences

photoluminescence brightness. Although there was no insight into control of surface reconstruction in the colloid literature, there were numerous discussions in the field of surface science on solid substrates. These inspired our group [14] to synthesize CdSe nanocrystals with photoluminescence quantum yields approaching unity by controlling the surface structure of the resulting nanocrystals (Fig. 9). Interestingly, in the same year, Weller's group [15] also reported that the photoluminescence brightness of semiconductor nanocrystals relied strongly on the growth conditions of the nanocrystals. The size of the brightest portion in an ensemble of semiconductor nanocrystals was found to be the one in equilibrium with the growth solution.

Another interesting goal of the synthetic chemistry of semiconductor nanocrystals, in consideration of their function as emitters, is optimizing the sizes achievable. If one wants to take advantage of their size-dependent emission colors, the resulting semiconductor nanocrystals should be in their quantum confinement size regime. For instance, if a synthetic scheme for InP nanocrystals only yielded particles greater than $20 \mathrm{~nm}$, it would not be possible to exploit the emission color tunability of these InP nanocrystals.

There are some other issues to be considered if one is to develop practical emissive materials. For example, the stability of the nanocrystals is an obvious concern. This is why core/shell semiconductor nanocrystals with a wide bandgap shell have attracted considerable attention in recent years $[47,48]$. Such core/shell nanocrystals could localize the photo-generated charges within the core and eliminate any photochemical and photophysical processes associated with the surface traps. Furthermore, the shell functions as a chemical barrier to protect the emissive core from possible chemical damage during processing as well as in device operation. As another example of the issues to be considered, any emissive materials must be eventually incorporated into a device. Thus, the processability and compatibility with the device design and fabrication technology must be considered. These issues might be considered as engineering issues in the future, but at present, they are still in the science domain as far as developing emissive materials for various applications is concerned.

When a specific application is in focus, the "function" consideration of colloidal nanocrystals often needs to be more specific as well. Still taking colloidal semiconductor nanocrystals for emissive materials as an example, let's concentrate on solidstate lighting. As pointed out previously, emissive nanocrystals could help to reduce the total energy consumption of human being by about $20 \%$, and thus have great potential to impact our life on a large scale.

Down-conversion of all (or a large portion) of high energy light-purple to blue, $\sim 400-460 \mathrm{~nm}$ wavelength range - from an efficient LED by emissive materials is the most promising technology for solidstate lighting at present. Inorganic phosphors are the current workhorse but their main problems include significant scattering loss by the phosphor powders, too broad and hard-to-tune photoluminescence spectra of the phosphors, and too low absorption cross-section of the phosphors. By looking at these problems, one may immediately see semiconductor nanocrystals can offer an excellent solution for this technology. The outstanding properties of semiconductor nanocrystals as far as their tunable and narrow photoluminescence and large absorption cross-section are concerned have been discussed in detail above (see Fig. 1 and the related text). As for

\section{量 Springer}


scattering, nanocrystals with proper surface ligand coatings should generally give negligible scattering (see Fig. 10(a) and 10(b), as an example). However, although semiconductor nanocrystals can overcome the intrinsic problems of inorganic phosphor powders, they are actually not ideal emitters for solid-state lighting if one thinks about their intrinsic bandgap photoluminescence.

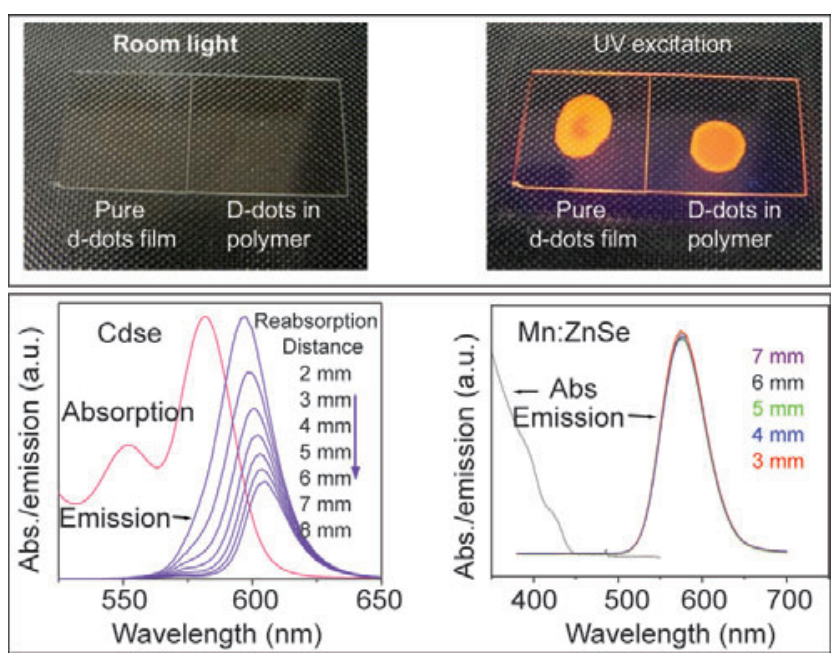

Figure 10 (a),(b) illustration of zero scattering nature of colloidal nanocrystals coated with ligands, with doped quantum dots (d-dots) as an example; (c),(d)comparison of the self-absorption of intrinsic bandgap photoluminescence of $\mathrm{CdSe}$ nanocrystals and dopant emission from Mn:ZnSe d-dots

The intrinsic bandgap photoluminescence of semiconductor nanocrystals has substantial overlap with its absorption (Fig. 2 and Fig. 10(c)). This becomes a huge problem when the optical density of the nanocrystal layer is high, which is exactly one of the requirements for the down-conversion for solidstate lighting. This is so because it is necessary to absorb most, if not all, of purple and blue emission from the LED. As shown in Fig. 10 (c), substantial self-quenching is observed for the bandgap photoluminescence from a semiconductor sample as the recording position is shifted away from the excitation point. To a lesser extent, another problem of bandgap emission of quantum dots is their intrinsic thermal sensitivity arising from the strong exciton-phonon coupling for typical semiconductor nanocrystals [49].

The above analysis indicates that it is unlikely that the bandgap photoluminescence may be utilized in solid-state lighting using down-conversion technology. Related to this, in fact, applications using the bandgap photoluminescence of semiconductor nanocrystals should be limited to semiconductor nanocrystals with a low optical density. For such applications, another type of emission from semiconductor nanocrystals, i.e., emission from the atomic dopant centers incorporated into the crystal lattices (dopant emission), may be an ideal solution.

When a quantum dot is doped with a certain type of impurity ion (which gives so-called d-dots), this may introduce some atomic states within the bandgap of the semiconductor nanocrystal. Upon an efficient energy transfer (or charge transfer) from the photo-excited semiconductor nanocrystal to the inner atomic states of a dopant center within the nanocrystal, usually involving the d-shell or f-shell of the transition metal dopant, radiative recombination occurs within these atomic states and emits a photon with somewhat reduced energy in comparison to the absorption edge. This has several important implications. Firstly, the absorption, or excitation, is realized by the semiconductor nanocrystal, which ensures a high absorption cross-section. Secondly, the dopant emission has a significantly red-shifted position from the absorption band of the nanocrystal, implying zero self-quenching of the emission caused by re-absorption, which contrasts sharply with the situation for bandgap emission (Figs. 10(c) and 10(d)). Thirdly, because the dopant emission occurs from inner atomic energy states of the dopant centers, it is not coupled with the phonons (or lattice vibration of the nanocrystals). As a result, dopant emission is much less sensitive to temperature $[49,50]$.

In comparison with intrinsic semiconductor nanocrystals, d-dots have been much less studied. In terms of the targeted function, dilute magnetic semiconductor nanocrystals for spintronics [51-54] have been the main focus to date. Difficulties in the synthesis of d-dots as high-performance emitters [50, 55-57] include doping every dot for color purity, greatly enhancing the emission efficiency, understanding and improving their stability, and achieving color tunability. These issues are thus 
the target problems for synthetic chemists to solve. The experimental results so far suggest that these issues are challenging but solvable [57]. At present, d-dot emitters covering most of the visible window with photoluminescence quantum yields as high as about $50 \%$ have already been demonstrated. Stability against thermal annealing [49] and chemical treatments [57] has also been verified for high quality d-dots. Furthermore, the d-dots intended for use as high-performance emitters are all based on nanocrystals without extremely toxic elements such as $\mathrm{Cd}, \mathrm{Hg}$, or $\mathrm{Pb}$. Such d-dots are currently being actively investigated by several companies for use in real life solid-state lighting devices.

In conclusion, colloidal nanocrystals have become highly important targets for synthetic chemistry because of their unique and diverse functions. It is thus critical for us to keep in mind what function is our goal when developing synthetic chemistry for a specific type of colloidal nanocrystals. This feature makes this new branch of synthetic chemistry, materials chemistry, a truly interdisciplinary field. In synthetic materials chemistry, a chemist with good synthetic training is critical but not sufficient. A deep understanding of the targeted functions, such as the physical principles underlying the functions, structure and function relationships, engineering requirements of the functional materials, and fabrication/operation variability of the functions of the materials, is crucial for developing truly useable functional materials.

\section{3. "Green" chemistry}

Green chemistry represents the awareness of the current physical boundaries of human beings, the earth. It is hard to find an accurate definition for this rich and diverse concept that is generally acceptable to everybody. However, its overall feature could be easily described as "Chemistry with environmental concern as one critical guideline". A common dispute is how harmless a chemical process should be before it is called a green chemical process.

When our group introduced $\mathrm{CdO}$ and other airstable cadmium compounds to replace dimethyl cadmium as the cadmium precursors for synthesis of high quality CdSe nanocrystals $[58,59]$, we claimed these methods implied a significant step toward green chemistry approaches for the synthesis of semiconductor nanocrystals. Quite some friends and coworkers laughed at us by saying "You are synthesizing CdSe nanocrystals. How can a product containing cadmium be green?". I have to agree that such arguments have a point. However, I personally like to adopt a broad viewpoint of green chemistry.

Yes, it is the ultimate goal of green chemistry to generate completely environmentally benign products. However, it is at least equally important for chemists to develop alternative routes to synthesize and process existing chemical products - colloidal nanocrystals in the context of this essay-with substantially reduced impact on the environment. One may further argue that, even for an environmentally benign product, the production process could pose a huge burden on our ecosystem. Taking a well known example, silicon crystals used in computer chips are environmentally benign but production of silicon-based computer chips needs a huge amount of energy, and raw materials amounting to a few thousand times more than the final products in mass. As a rough picture, production of one computer could generate thousands times more waste by mass - usually harmful-for our earth to contain.

In 2002, the author published a Concepts article in Chemistry-A European Journal on green chemical approaches toward semiconductor nanocrystals [7]. Below, let's revisit this topic using the same class of colloidal nanocrystals to illustrate how synthetic chemistry of colloidal nanocrystals could evolve according to suggested green chemistry guidelines.

\subsection{Alternative routes}

Alternative routes for the synthesis of colloidal nanocrystals are still the main focus of green chemistry developments in the field. Although high quality colloidal nanocrystals are new materials synthesized in new ways, there is still great room for improving their synthetic chemistry in terms of reducing environmental impact.

The introduction of $\mathrm{CdO}$ and air-stable cadmium precursors for the synthesis of CdSe nanocrystals 
in 2001 was considered as the first step towards "greener" methods for the synthesis of high quality semiconductor nanocrystals [58]. The original synthesis of high quality CdSe nanocrystals, which was initiated by the Brus group [23] and finalized by the Bawendi group [24] with some additional modifications by the Alivisatos group [9, 16], relied on the injection of a cold precursor solution containing dimethylcadmium mixed with seleniumtributylphosphine into very hot trioctylphosphine oxide solvent. The reaction temperature was about 300$360{ }^{\circ} \mathrm{C}$ in the finalized version. Dimethylcadmium is extremely toxic, dangerous, and expensive, as pointed out above, and its replacement by $\mathrm{CdO}$ or other air-stable cadmium precursor made the synthesis practicable in a regular synthetic laboratory.

However, the new versions of synthesis for CdSe nanocrystals introduced in 2001 still had some scope to be substantially greener. The elimination of tributylphosphine (or other organophosphines) and the use of non-injection methods basically removed all strongly air-sensitive chemicals from the synthesis and further simplified the synthetic procedure [29].

One of the most recent advances making the synthesis of CdSe nanocrystals even greener is the reduction of the reaction temperature from around $300{ }^{\circ} \mathrm{C}$ to about $150{ }^{\circ} \mathrm{C}$ (Fig. 11(a)) [20]. Upon studying the reaction ligand dynamics on the surface of CdSe nanocrystals, it was found that the high temperature requirement imposed in the past was mainly due to the surface ligand dynamics. The slow dynamics of the chosen surface ligands on the nanocrystals meant that the growth rate of the nanocrystal was very slow unless the reaction temperatures were high. The research further identified that the surface ligand dynamics for relatively weak ligands can be roughly correlated with the boiling point of the ligands. Thus, by using a relatively short aliphatic amine (such as dodecylamine) in place of the longer chain ones (such as octadecylamine) originally used, the required reaction temperature could be reduced from around $300{ }^{\circ} \mathrm{C}$ to about $150{ }^{\circ} \mathrm{C}$ without compromising the quality of the nanocrystals (Fig. 11(a)). Reduction of reaction temperature not only gives safer and simpler production procedures, but also involves lower
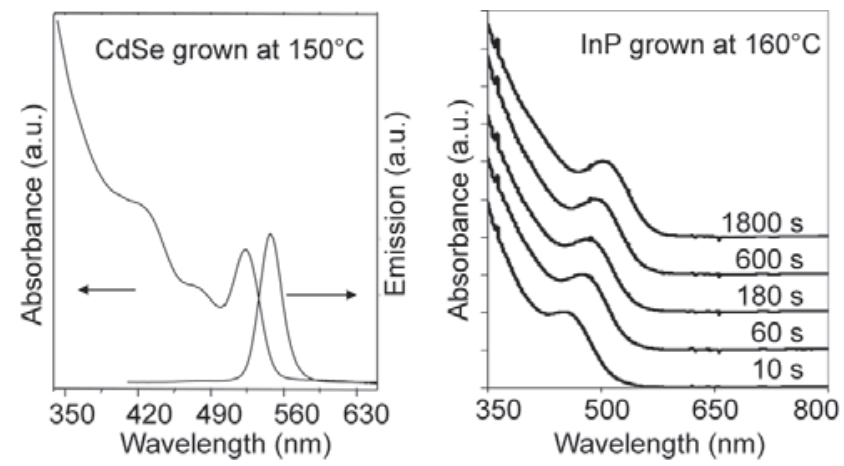

Figure 11 Reducing reaction temperature by using ligands with low boiling points for synthesis of $\mathrm{CdSe}(\mathrm{a})$ and $\ln \mathrm{P}(\mathrm{b})$ nanocrystals

energy consumption. It is thus of significance for green chemistry.

It should be emphasized that any alternative more environmentally-friendly route has to yield products with similar quality -if not better - to that of the original synthesis. Furthermore, countless examples illustrate that alternative routes are often discovered on the basis of a new insight into the mechanism of a specific synthetic system. This means that "greener" often also means more "rational" at the same time. For example, the reduction in reaction temperature discussed in the above paragraph was extended to the synthesis of other types of semiconductor nanocrystals, such as the III-V type (Fig. 11(b)) $[26,60]$. Such a procedure can be established as a new synthetic concept because the underlying chemical principle i.e., that the reaction temperature is determined by the surface ligand dynamics, has been established through quantitative and systematic studies of the original system.

\subsection{Greener products}

Greener products are certainly desirable if comparable synthetic approaches can be devised. As for semiconductor nanocrystals, the current workhorse is still the CdSe nanocrystal system. There is no doubt that CdSe nanocrystals cannot be widely used as emitters because of the intrinsic toxicity of cadmium (Table 1). The most optimistic view would be that cadmium-containing semiconductor nanocrystals might have some limited applications in some very special cases, such as in R\&D. This means that synthetic chemists working in the field of colloidal nanocrystals 
must seek "greener products" to replace CdSe and related nanocrystals, including $\mathrm{CdTe}, \mathrm{CdS}$, and the corresponding core/shell ones.

Table 1 Class A and Class B elements commonly encountered in nanocrystals summarized from the data published by Electronic Industry Alliance (http://www.eia.org/)

\begin{tabular}{l|l}
\hline $\begin{array}{l}\text { Class A (intentional inclusion is } \\
\text { prohibited) }\end{array}$ & $\begin{array}{l}\text { Class B }(<0.1 \% \text { by mass in } \\
\text { product is allowed) }\end{array}$ \\
\hline $\begin{array}{l}\mathrm{Cd} \text { (in all forms), } \mathrm{Hg} \text { (in all } \\
\text { forms), } \mathrm{Pb} \text { (in all forms), } \mathrm{Sn} \\
\text { (certain organometallic forms) }\end{array}$ & $\begin{array}{l}\mathrm{Se} \text { (in all forms), As (in all forms), } \\
\mathrm{Sb} \text { (in all forms), Be (in all forms) }\end{array}$ \\
\hline
\end{tabular}

According to the Electronic Industry Alliance (http:/ / www.eia.org/), the allowed tolerance level of elements in new products differs from one element to another. Table 1 summarizes the environmental limits for some of the elements closely related to development of colloidal semiconductor nanocrystals for electronics, optics, and optoelectronics. It should be pointed out that, however, other applications - such as those in medical fields - may require additional standards.

Cadmium is listed as a Class A element in the industrial standards (Table 1), and has zero tolerance in today's environmental policy unless it is introduced unintentionally; in the latter case, the allowed limit is $<100 \mathrm{ppm}$. Replacements for cadmium-based semiconductor nanocrystals must not contain any other Class A elements and the performance of the nanocrystals must be comparable to the existing ones. Therefore, any nanocrystals containing $\mathrm{Hg}$ and $\mathrm{Pb}$ should not be considered at all. This also implies that, unless there is special significance, colloidal nanocrystals containing $\mathrm{Cd}$, $\mathrm{Hg}$, and $\mathrm{Pb}$ are barely interesting as functional materials.

As discussed above, d-dots based on $\mathrm{ZnSe}$ and ZnS nanocrystals as the hosts (Fig. 10), are potentially ideal emitters for one of the major applications of semiconductor nanocrystals, namely solid-state lighting. All of the elements involved so far, $\mathrm{Zn}, \mathrm{Se}$, $\mathrm{S}, \mathrm{Cu}$, and $\mathrm{Mn}$ are acceptable under the industrial standards according to Table 1. Certainly, however, it would be of interest to replace Se (the only Class B element) by another greener element.
Although d-dots based on zinc chalcogenides are ideal for solid-state lighting as measured by their function, they might not always be the best emitters for other applications. For example, for in vivo biomedical imaging [61] (Fig. 12(b)), it is desirable to have both excitation and emission in the near infrared window because of the transparent nature of biological tissues in this wavelength window. It will not be possible to realize significant absorption in the near infrared with zinc chalcogenide nanocrystals. For this and some other applications, intrinsic bandgap emission should work better than the dopant emission offered by d-dots. This is why development of highly emissive and stable ZnSe (covering near UV, purple, and blue) [62], InP (covering blue to deep red) (Fig. 12) [60], and InAs (covering the near infrared) [26] intrinsic quantum dots is important as well. It should be noticed that, although all of these intrinsic nanocrystals meet the current industrial standards to a large extent, Class B elements (Se and As) and some other heavy metals (In and Zn, especially In) are still included, which might not be perfect for medical applications.
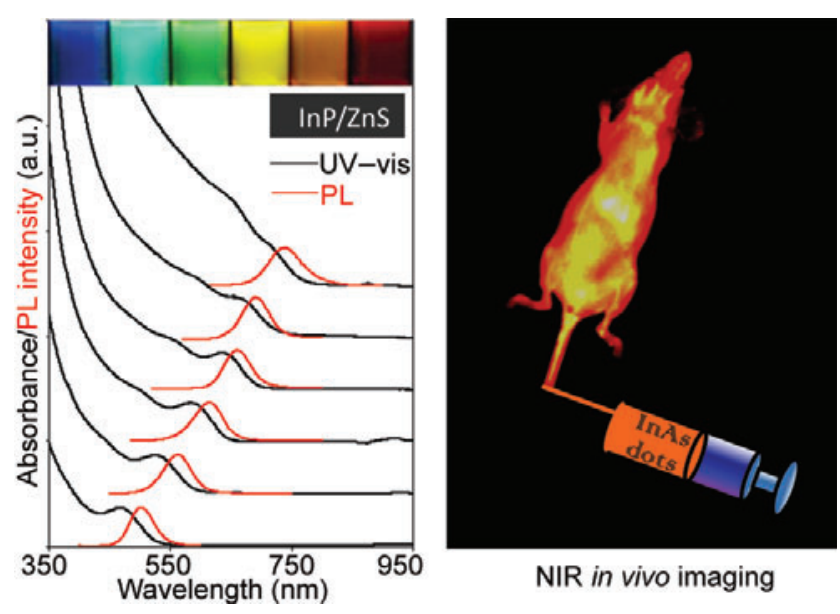

Figure 12 Non-cadmium quantum dots covering most of the visible window (InP dots, (a)) and the near infrared (NIR) window for in vivo imaging (InAs dots, (b))

\subsection{The overall cost of a product}

The overall cost of a product with a required function has become a rough measure of how green a chemical process and its products are. This measure is not scientific. Instead, we have to rely on business leaders 
to decide the future of industry. Generally, if a process uses some highly toxic and dangerous chemicals / procedures, the costs of waste disposal and equipment will be substantial. Similarly, if a product has a huge adverse impact on the environment, it will immediately lower its competitive edge and be expensive to use and market it.

This non-scientific rule may also be applied in laboratory practices. If one works on mercuryrelated nanocrystals, the waste disposal (at least in advanced countries such as USA) will become excessively expensive. When a reaction can be performed with a small volume, on a scale with 4-5 $\mathrm{mL}$ of solvent as is usually the case in my laboratory, it does not make sense to increase the volume substantially unless the synthesis has a clear need for scale-up. If a synthesis yields high quality nanocrystals with minimum purification, it will always be cheaper and greener.

\section{Closing remarks}

Synthetic chemistry of colloidal nanocrystals is undoubtedly an important subject in modern chemistry. However, its importance at present is mainly due to its potential products, functional materials based on colloidal nanocrystals. As a comparison, synthetic organic chemistry is truly a mature field in basic science, with its own fundamental framework and unique characteristics, which can be taught to many generations of junior scientists in the form of broadly applicable professional skills and specialized thinking. For instance, without modern organic chemistry, the polymer and pharmaceutical industries would not be possible.

One may argue that the synthetic chemistry of colloidal nanocrystals may one day become a field similar to synthetic organic chemistry because this new subject creates an equally diverse class of new (inorganic) substances just as organic synthetic chemistry does for organic compounds. This author likes this optimistic picture but it is yet to be proven. At present, our main mission is still to demonstrate feasibilities and discover new avenues for exploiting the special properties of colloidal nanocrystals in many real life applications. The faster we realize one type of real practical application, the more secure the future of synthetic chemistry of colloidal nanocrystals as a true fundamental science field will be. Hopefully, along with these critically important demonstrations and discoveries of functional materials, the synthetic chemistry of colloidal nanocrystals will rapidly expand its scope and deepen its roots as a new subject in fundamental science.

Therefore, although this essay proposes three basic rules for developing the synthetic chemistry of colloidal nanocrystals, the most important one at this moment is "functional". The fundamental knowledge associated with "rational" synthesis might be equally important for its significance in understanding crystallization, solution surface chemistry, and developing colloidal nanocrystal synthesis. "Green" basically tells us that choices of chemicals and processes allowed by our fragile environment will play a limiting role in our creation of functional materials.

In summary, the demands for functional materials come from everyday life. Engineers, working with business leaders and government policy makers, translate such needs into engineering requirements. Synthetic chemists must listen carefully to understand the whole picture. In this way, we will ensure we are not just

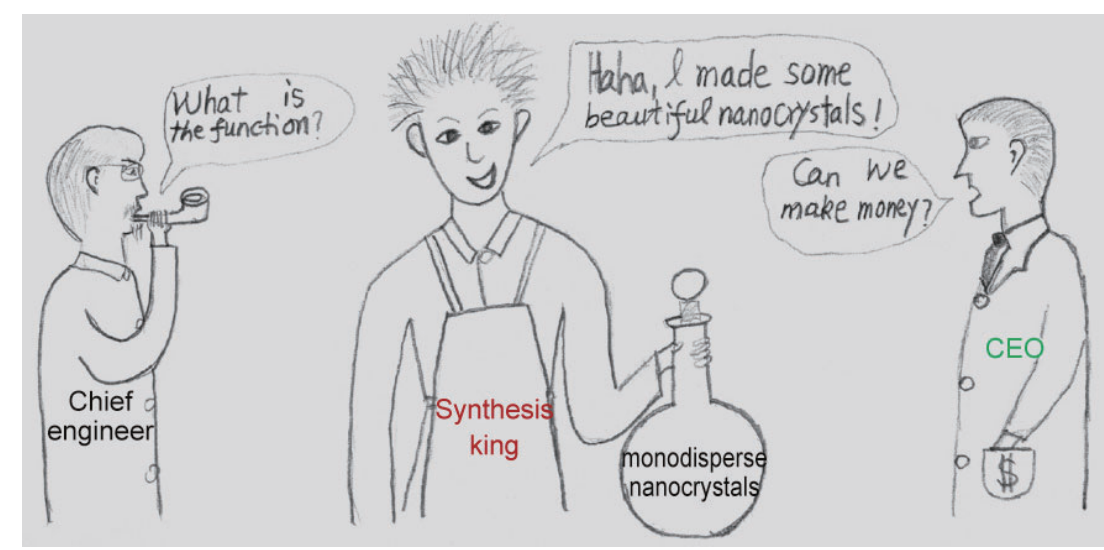

Figure 13 Synthetic chemists should not concentrate on nanocrystals for their "pretty looks", but rather create them with functions giving rise to applications in everyday life by closel collaboration with engineers and others 
synthesizing something which looks pretty but instead create colloidal nanocrystals which work effectively in the real world (Fig. 13).

\section{Acknowledgements}

Financial support from the National Science Foundation is acknowledged. Ms. Michelle Peng helped in preparing Fig. 13.

\section{References}

[1] Armaroli, N.; Balzani, V. The future of energy supply: Challenges and opportunities. Angew. Chem. Int. Ed 2007, 46, 52-66.

[2] Brus, L. E. A simple model for the ionization potential, electron affinity, and aqueous redox potentials of small semiconductor crystallites. J. Chem. Phys. 1983, 79, 5566-71.

[3] Peng, Z. A.; Peng, X. Nearly monodisperse and shapecontrolled CdSe nanocrystals via alternative routes: Nucleation and growth. J. Am. Chem. Soc. 2002, 124, 3343-3353.

[4] Turkevich, J. Collodial gold. Part I. Gold Bull. 1985, 18, 86-91.

[5] Mullin, J. W. Crystallization, 3nd Edn. ButterworthHeinemann: Oxford, 1997.

[6] Oxtoby, D. W. Nucleation of first-order phase transitions. Acc. Chem. Res. 1998, 31, 91-97.

[7] Peng, X. G. Green chemical approaches toward highquality semiconductor nanocrystals. Chem. Eur. J. 2002, 8, 334-339.

[8] Murray, C. B.; Sun, S. H.; Gaschler, W.; Doyle, H.; Betley, T. A.; Kagan, C. R. Colloidal synthesis of nanocrystals and nanocrystal superlattices. IBM J. Res. Dev. 2001, 45, 4756.

[9] Peng, X. G.; Manna, L.; Yang, W. D.; Wickham, J.; Scher, E.; Kadavanich, A.; Allvisatos, A. P. Shape control of CdSe nanocrystals. Nature 2000, 404, 59-61.

[10] Thessing, J.; Qian, J. H.; Chen, H. Y.; Pradhan, N.; Peng, X. G. Interparticle influence on size/size distribution evolution of nanocrystals. J. Am. Chem. Soc. 2007, 129, 2736-2737.

[11] Alivisatos, A. P. Semiconductor clusters, nanocrystals, and quantum dots. Science 1996, 271, 933-937.

[12] Brus, L. E. Electron-electron and electron-hole interactions in small semiconductor crystallites: The size dependence of the lowest excited electronic state. J. Chem. Phys. 1984, 80, 4403-4409.

[13] Yu, W. W.; Qu, L. H.; Guo, W. Z.; Peng, X. G. Experimental determination of the extinction coefficient of CdTe, CdSe, and CdS nanocrystals. Chem. Mater. 2003, 15, 2854-2860.

[14] Qu, L. H.; Peng, X. G. Control of photoluminescence properties of $\mathrm{CdSe}$ nanocrystals in growth. J. Am. Chem. Soc. 2002, 124, 2049-2055.

[15] Talapin, D. V.; Rogach, A. L.; Shevchenko, E. V.; Kornowski, A.; Haase, M.; Weller, H. Dynamic distribution of growth rates within the ensembles of colloidal II-VI and III-V semiconductor nanocrystals as a aactor governing their photoluminescence efficiency. J. Am. Chem. Soc. 2002, 124, 5782-5790.

[16] Peng, X. G.; Wickham, J.; Alivisatos, A. P. Kinetics of II$\mathrm{VI}$ and III-V colloidal semiconductor nanocrystal growth: "Focusing" of size distributions. J. Am. Chem. Soc. 1998, 120, 5343-5344.

[17] Chen, Y. F.; Kim, M.; Lian, G. D.; Johnson, M. B.; Peng, $X$. G. Side reactions in controlling the quality, yield, and stability of high quality colloidal nanocrystals. J. Am. Chem. Soc. 2005, 127 (38), 13331-13337.

[18] Holland, G. P.; Sharma, R.; Agola, J. O.; Amin, S.; Solomon, V. C.; Singh, P.; Buttry, D. A.; Yarger, J. L. NMR characterization of phosphonic acid capped $\mathrm{SnO}_{2}$ nanoparticles. Chem. Mater. 2007, 19, 2519-2526.

[19] Ji, X. H.; Copenhaver, D.; Sichmeller, C.; Peng, X. G. Ligand bonding and dynamics on colloidal nanocrystals at room temperature: The case of alkylamines on CdSe nanocrystals. J. Am. Chem. Soc. 2008, 130, 5726-5735.

[20] Pradhan, N.; Reifsnyder, D.; Xie, R. G.; Aldana, J.; Peng, X. G. Surface ligand dynamics in growth of nanocrystals. J. Am. Chem. Soc. 2007, 129, 9500-9509.

[21] Chen, Y. F.; Johnson, E.; Peng, X. G. Formation of monodisperse and shape-controlled $\mathrm{MnO}$ nanocrystals in non-injection synthesis: Self-focusing via ripening. J. Am. Chem. Soc. 2007, 129, 10937-10947.

[22] Ji, X. H.; Song, X. N.; Li, J.; Bai, Y. B.; Yang, W. S.; Peng, X. $G$. Size control of gold nanocrystals in citrate reduction: The third role of citrate. J. Am. Chem. Soc. 2007, 129, 13939-13948.

[23] Steigerwald, M. L.; Brus, L. E. Semiconductor crystallites: A class of large molecules. Acc. Chem. Res. 1990, 23, 183-188. 
[24] Murray, C. B.; Norris, D. J.; Bawendi, M. G. Synthesis and characterization of nearly monodisperse $\mathrm{CdE}$ ( $\mathrm{E}=$ sulfur, selenium, tellurium) semiconductor nanocrystallites. J. Am. Chem. Soc. 1993, 115, 8706-15.

[25] Sugimoto, T. Preparation of monodispersed colloidal particles. Adv. Colloid Interface Sci. 1987, 28, 65-108.

[26] Xie, R. G.; Peng, X. G. Synthetic scheme for high-quality InAs nanocrystals based on self-focusing and one-pot synthesis of InAs-based core-shell nanocrystals. Angew. Chem. Int. Ed. 2008, 47, 7677-7680.

[27] Cumberland, S. L.; Hanif, K. M.; Javier, A.; Khitrov, G. A.; Strouse, G. F.; Woessner, S. M.; Yun, C. S. Inorganic clusters as single-source precursors for preparation of CdSe, ZnSe, and CdSe/ZnS nanomaterials. Chem. Mater. 2002, 14, 1576-1584.

[28] Qu, L. H.; Yu, W. W.; Peng, X. G. In situ observation of the nucleation and growth of CdSe nanocrystals. Nano Lett. 2004, 4, 465-469.

[29] Yang, Y. A.; Wu, H. M.; Williams, K. R.; Cao, Y. C. Synthesis of CdSe and CdTe nanocrystals without precursor injection. Angew. Chem. Int. Ed. 2005, 44, 6712-6715

[30] Murray, C. B.; Kagan, C. R.; Bawendi, M. G. Synthesis and characterization of monodisperse nanocrystals and close-paked nanocrystal assemblies. Annu. Rev. Mater. Sci. 2000, 30, 545-610.

[31] Pacholski, C.; Kornowski, A.; Weller, H. Self-assembly of ZnO: From nanodots to nanorods. Angew. Chem. Int. Ed. 2002, 41, 1188-1191.

[32] Tang, Z.; Kotov, N. A.; Giersig, M. Science 2002, 297, 237-240.

[33] Cho, K. -S.; Talapin, D. V.; Gaschler, W.; Murray, C. $B$. Designing PbSe nanowires and nanorings through oriented attachment of nanoparticles. J. Am. Chem. Soc. 2005, 127, 7140-7147.

[34] Narayanaswamy, A.; Xu, H. F.; Pradhan, N.; Peng, X. $G$. Crystalline nanoflowers withdifferent chemical compositions and physical properties grown by limited ligand protection. Angew. Chem. Int. Ed. 2006, 45, 5361-5364.

[35] Yu, W. W.; Peng, X. G. Formation of high-quality CdS and other II-VI semiconductor nanocrystals in noncoordinating solvents: Tunable reactivity of monomers. Angew. Chem., Int. Edn. 2002, 41, 2368-2371.

[36] Peng, Z. A.; Peng, X. G. Mechanisms of the shape evolution of CdSe nanocrystals. J. Am. Chem. Soc. 2001,
123, 1389-1395.

[37] Bruchez, M., Jr.; Moronne, M.; Gin, P.; Weiss, S.; Alivisatos, A. P. Semiconductor nanocrystals as fluorescent biological labels. Science 1998, 281, 2013-2016.

[38] Chan, W. C. W.; Nile, S. M. Quantum dot bioconjugates for ultrasensitive nonisotopic detection. Science 1998, 281, 2016-2018

[39] Greenham, N. C.; Peng, X.; Alivisatos, A. P. Charge separation and transport in conjugated-polymer/ semiconductor-nanocrystal composites studied by photoluminescence quenching and photoconductivity. Phys. Rev. B 1996, 54, 17628-17637.

[40] Munro, A. M.; Plante, I. J. -L.; Ng, M. S.; Ginger, D. S. Quantitative study of the effects of surface ligand concentration on CdSe nanocrystal photoluminescence. J. Phys. Chem. C 2007, 111, 6220-6227.

[41] Bullen, C.; Mulvaney, P. The effects of chemisorption on the luminescence of CdSe quantum dots. Langmuir 2006, 22, 3007-3013

[42] Wang, Y. A.; Li, J. J.; Chen, H. Y.; Peng, X. G. Stabilization of inorganic nanocrystals by organic dendrons. J. Am. Chem. Soc. 2002, 124, 2293-2298.

[43] Heaven, M. W.; Dass, A.; White, P. S.; Holt, K. M.; Murray, R. W. Crystal structure of the gold nanoparticle $\left[\mathrm{N}\left(\mathrm{C}_{8} \mathrm{H}_{17}\right)_{4}\right]\left[\mathrm{Au}_{25}\left(\mathrm{SCH}_{2} \mathrm{CH}_{2} \mathrm{Ph}\right)_{18}\right]$. J. Am. Chem. Soc. 2008, 130, 3754-3755.

[44] Jana, N. R.; Peng, X. G. Single-phase and gram-scale routes toward nearly monodisperse $\mathrm{Au}$ and other noble metal nanocrystals. J. Am. Chem. Soc. 2003, 125, 14280 $-14281$.

[45] Steigerwald, M. L.; Brus, L. E. Synthesis, stabilization, and electronic structure of quantum semiconductor nanoclusters. Annu. Rev. Mater. Sci. 1989, 19, 471-495.

[46] Katari, J. E. B.; Colvin, V. L.; Alivisatos, A. P. X-ray photoelectron spectroscopy of CdSe nanocrystals with applications to studies of the nanocrystal surface. J. Phys. Chem. 1994, 98, 4109-4117.

[47] Hines, M. A.; Guyot-Sionnest, P. Second harmonic generation studies of methylene blue orientation at silica surfaces. J. Phys. Chem. 1996, 100, 468-471.

[48] Li, J. J.; Wang, Y. A.; Guo, W. Z.; Keay, J. C.; Mishima, T. D.; Johnson, M. B.; Peng, X. G. Large-scale synthesis of nearly monodisperse $\mathrm{CdSe/CdS}$ core/shell nanocrystals using air-stable reagents via successive ion layer adsorption and reaction. J. Am. Chem. Soc. 2003, 125, 12567-12575. 
[49] Pradhan, N.; Battaglia, D. M.; Liu, Y.; Peng, X. Efficient, stable, small, and water-soluble doped ZnSe nanocrystal emitters as non-cadmium biomedical labels. Nano Lett. 2007, 7, 312-317.

[50] Pradhan, N.; Goorskey, D.; Thessing, J.; Peng, X. G. An alternative of CdSe nanocrystal emitters: Pure and tunable impurity emissions in ZnSe nanocrystals. J. Am. Chem. Soc. 2005, 127, 17586-17587.

[51] Norris, D. J.; Yao, N.; Charnock, F. T.; Kennedy, T. A. High-quality manganese-doped ZnSe nanocrystals. Nano Lett. 2001, 1, 3-7.

[52] Radovanovic, P. V.; Gamelin, D. R. Electronic absorption spectroscopy of cobalt ions in diluted magnetic semiconductor quantum dots: Demonstration of an isocrystalline core/shell synthetic method. J. Am. Chem. Soc. 2001, 123, 12207-12214.

[53] Hanif, K. M.; Meulenberg, R. W.; Strouse, G. F. Magnetic ordering in doped $\mathrm{Cd}_{1-x} \mathrm{CO}_{x} \mathrm{Se}$ diluted magnetic quantum dots. J. Am. Chem. Soc. 2002, 124, 11495-11502.

[54] Schwartz, D. A.; Norberg, N. S.; Nguyen, Q. P.; Parker, J. M.; Gamelin, D. R. Magnetic quantum dots: Synthesis, spectroscopy, and magnetism of $\mathrm{CO}^{2+}$ - and $\mathrm{Ni}^{2+}$-doped ZnO nanocrystals. J. Am. Chem. Soc. 2003, 125, 1320513218.

[55] Bhargava, R. N.; Gallagher, D.; Hong, X.; Nurmikko, A. Optical properties of manganese-doped nanocrystals of ZnS. Phys. Rev. Lett. 1994, 72, 416-419.
[56] Yang, Y. G.; Chen, O.; Angerhofer, A.; Cao, Y. C. Radial-position-controlled doping in CdS/ZnS core/Shell nanocrystals. J. Am. Chem. Soc. 2006, 128, 1242812429.

[57] Pradhan, N.; Peng, X. G. Efficient and color-tunable Mn-doped ZnSe nanocrystal emitters: control of optical performance via greener synthetic chemistry. J. Am. Chem. Soc. 2007, 129, 3339-3347.

[58] Peng, Z. A.; Peng, X. G. Formation of high-quality CdTe, CdSe, and CdS nanocrystals using $\mathrm{CdO}$ as precursor. J. Am. Chem. Soc. 2001, 123, 183-184.

[59] Qu, L. H.; Peng, Z. A.; Peng, X. G. Alternative routes toward high quality CdSe nanocrystals. Nano Lett. 2001, 1, 333-337.

[60] Xie, R. G.; Battaglia, D.; Peng, X. G. Colloidal InP nanocrystals as efficient emitters covering blue to nearinfrare. J. Am. Chem. Soc. 2007, 129, 15432-15433.

[61] Xie, R. G.; Chen, K.; Chen, X. Y.; Peng X. G. InAs/InP/ ZnSe core/shell/shell quantum dots as near-infrared emitters: Bright, narrow-band, non-cadmium containing, and biocompatible. Nano Res. 2008, 1, 457-464.

[62] Li, L. S.; Pradhan, N.; Wang, Y. J.; Peng, X. G. High quality ZnSe and ZnS nanocrystals formed by activating zinc carboxylate precursors. Nano Lett. 2004, 4, 22612264. 